\title{
TEM investigation of the microstructural evolution during nanoindentation of $\mathrm{NiTi}$
}

\author{
J. Pfetzing a, M.F.-X. Wagner, T. Simon, A. Schaefer, Ch. Somsen, G. Eggeler \\ Ruhr-University Bochum, Institute for Materials, 44780 Bochum, Germany
}

\begin{abstract}
The continuous measurement of loads and displacements during nanoindentation of shape memory alloys allows a detailed investigation of pseudoelasticity on the nano-scale. However, the resulting load-displacement data simultaneously reflect several elastic and inelastic deformation processes (i.e., stress-induced martensitic transformation, plastic deformation). In the present study, we perform microstructural investigations in order to analyze the complex interactions between these mechanisms in a Ni-rich alloy. The microstructures below indents from experiments with different maximum indentation loads are characterized post-mortem by transmission electron microscopy. For small maximum indentation loads, load-displacement data exhibit considerable pseudoelastic recovery, whereas higher indentation loads are associated with a more pronounced residual deformation after unloading. These differences are clearly related to microstructural changes below the indenter tip: While both stressinduced martensitic transformation and dislocation slip occur in general, higher maximum loads are associated with an increase in plastic deformation. The corresponding higher dislocation densities impede the reverse transformation to austenite. Stabilized martensite can be observed directly below the surface for high indentation loads. Our results show that nanoindentation of NiTi allows a systematic analysis of the interaction of plastic deformation and stress induced transformation into martensite.
\end{abstract}

\section{Introduction}

NiTi shape memory alloys (NiTi SMA) are used in various small scale engineering and medical applications, for instance stents or micro electro-mechanical systems [1]. Proper characterization of the local mechanical properties of NiTi SMA is essential for the development and application of such devices. Nanoindentation is an attractive tool for the analysis of the mechanical behavior of small sample volumes [2-5]. During nanoindentation experiments, the low loads and small displacements are measured continuously, and typical load-displacement curves are recorded. Recent studies on the nanoindentation behavior of NiTi focused on characterizing the mechanical properties and transformation behavior by analyzing load-displacement data [610] and/or the residual surface features after indentation [10-15]. A widely used technique to quantify the amount of pseudoelastic recovery during nanoindentation testing is calculating the ratio of the remnant displacement $h_{\text {rem }}$ and the maximum displacement $\mathrm{h}_{\max }$ from load-displacement curves $[6,16,17]$ :

$$
\mathrm{RDR}=\frac{\mathrm{h}_{\mathrm{rem}}}{\mathrm{h}_{\text {max }}} .
$$

Pseudoelastic recovery is characterized by low values of the remnant displacement ratio (RDR), whereas large RDR-values are associated with a more pronounced effect of plastic deformation. Although load-displacement curves and RDR-values yield qualitative information regarding the deformation mechanisms during indentation, a detailed microstructural analysis is necessary to understand the interaction of elastic, plastic and pseudoelastic deformation below the indenter tip. Such approaches, which involve careful transmission electron microscopy (TEM) observations of the deformed microstructures, have recently been applied to conventional, elasto-plastic materials $[18,19]$. In an earlier work [20], we performed a nanoindentation experiment with a sharp (Berkovich) indenter tip on a thin NiTiFe foil. Subsequent TEM observations of the indented region showed martensite stabilized by dislocations directly below the indent. However, the mechanical results (load-displacement data)

a e-mail: Janine.Pfetzing@rub. de

This is an Open Access article distributed under the terms of the Creative Commons Attribution-Noncommercial License (http://creativecommons.org/licenses/by-nc/3.0/), which permits unrestricted use, distribution, and reproduction in any noncommercial medium, provided the original work is properly cited. 
could not directly be related to the different microstructural deformation processes because of various experimental difficulties, e.g., bending of the foil, effects of glue and sample holder. Moreover, different investigations $[6,16]$ demonstrated that spherical indenter tips are considerably better suited to document pseudoelastic material behavior. In the present study, we investigate the deformed microstructures in pseudoelastic bulk NiTi after indentation with a spherical indenter tip and two different maximum indentation loads. The TEM observations, which are in line with our previous results, are qualitatively discussed considering the interaction of plastic deformation and the stress-induced formation of martensite.

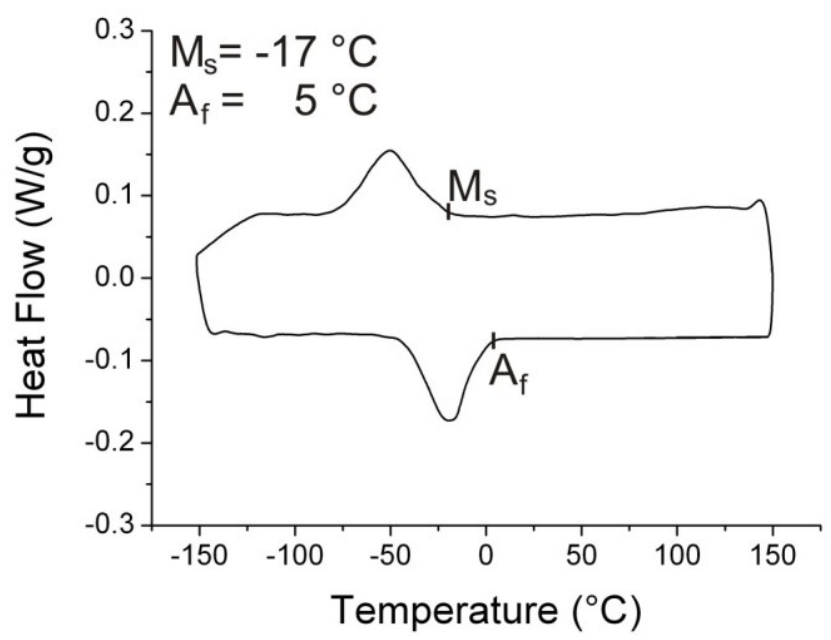

Fig. 1. The DSC-Diagram for the solution annealed, pseudoelastic NiTi indicates a single-step transformation from B2 to B19' during cooling and the corresponding reverse transformation during heating.

\section{Experimental}

Binary NiTi rods with a nominal Ni-concentration of 50.9 at.\% were obtained from Euroflex G. Rau GmbH (Pforzheim, Germany). The as-received material was solution annealed at $850{ }^{\circ} \mathrm{C}$ for $1 \mathrm{~h}$ followed by water quenching. The phase transformation temperatures were determined by differential scanning calorimetry (DSC) using a Thermal Analysis $2920 \mathrm{CE}$ instrument in a temperature range from -150 to $150{ }^{\circ} \mathrm{C}$ with a heating/cooling rate of $10 \mathrm{~K} / \mathrm{min}$. The solution annealed material exhibits a single-step transformation from austenite (B2) to martensite (B19') during cooling and a reverse transformation from B19' to B2 during heating, Fig. 1. As the austenite finish temperature, $\mathrm{A}_{\mathrm{f}} \approx 5^{\circ} \mathrm{C}$, is well below room temperature, the NiTi specimen is expected to be pseudoelastic at the testing temperature used here.

Samples for nanoindentation were carefully mechanically ground and polished. Final polishing was performed on a vibratory polisher (Buehler, Vibromet 2) using a colloidal silica suspension. Nanoindentation experiments were performed using a MTS Nanoindenter XP with a spherical diamond indenter tip (radius $\mathrm{R}=5 \mu \mathrm{m}$ ). The indentation procedure was performed with a MTS standard method (XP Basic) with a loading rate of $1 \mathrm{mN} / \mathrm{s}$.

For the microstructural investigation after indentation, TEM samples were prepared using the focused ion beam (FIB) technique (details are given in [18, 21-23]). To mark the location of the indented surface for FIB preparation, the indents selected for subsequent microstructural analysis (with relatively small maximum loads of 10 and $30 \mathrm{mN}$ ) were placed between two marking indents (with larger indentation depths for easier identification in the nanoindenter's optical microscope). The FIB lamellae were prepared with a Dual Beam FEI, type Quanta 200 3D. To prevent damage by ions, a protective tungsten layer with a thickness of about $1 \mu \mathrm{m}$ was deposited. Subsequently, the surface below and above this layer was removed gradually up to a lamella thickness of $\sim 600 \mathrm{~nm}$. Fig. 2 shows an SEM micrograph of the lamella taken during FIB preparation. The final polishing of the lamella resulted in a thickness of $\sim 150 \mathrm{~nm}$. The TEM investigations were performed on a Philips CM 20 instrument operating at $200 \mathrm{kV}$.

\section{Results and Discussion}

The load-displacement data associated with the nanoindentation measurements in bulk pseudoelastic NiTi are shown in Fig. 3. Indentation with a maximum load of $10 \mathrm{mN}$ (Fig. 3a) results in a typical pseudoelastic loaddisplacement curve that exhibits a small mechanical hysteresis. During unloading, pseudoelastic recovery is almost complete and only a small remnant displacement of $\sim 20 \mathrm{~nm}$ is observed (this corresponds to an RDR- 
value of $10.6 \%$ ). The load-displacement curve for indentation with a maximum load of $30 \mathrm{mN}$ (Fig. $4 \mathrm{~b}$ ) exhibits a larger remnant displacement of $160 \mathrm{~nm}(\mathrm{RDR}=30.7 \%)$. To identify the microstructural changes and deformation mechanisms in detail, we performed TEM investigations of the microstructure directly below the indented surface. Typical TEM observations of the microstructure below the $10 \mathrm{mN}$ indent are summarized in Fig. 4. The bright field image (Fig. 4a) shows an austenitic microstructure and a high dislocation density in an approximately semi-spherical region (with a radius of about $3 \mu \mathrm{m}$ ) below the indented surface. The orange circles in Fig. 5 indicate the regions where diffraction patterns were taken. A representative pattern with spots of the B2 phase of type $<1 \overline{1} 0>$ is shown in Fig. 4b. The red boxes in Fig. 4a indicate two regions where the dislocation substructures were investigated in more detail. Fig. 4c shows a high dislocation density about $200 \mathrm{~nm}$ below the surface (region 1 in Fig. 4a). With increasing distance from the surface, the dislocation density decreases, Fig. 4d (region 2 in Fig. 4a). As the stress-induced martensite is completely transformed back to austenite after unloading, the small residual deformation observed in the load-displacement curves is clearly due to dislocation plasticity.

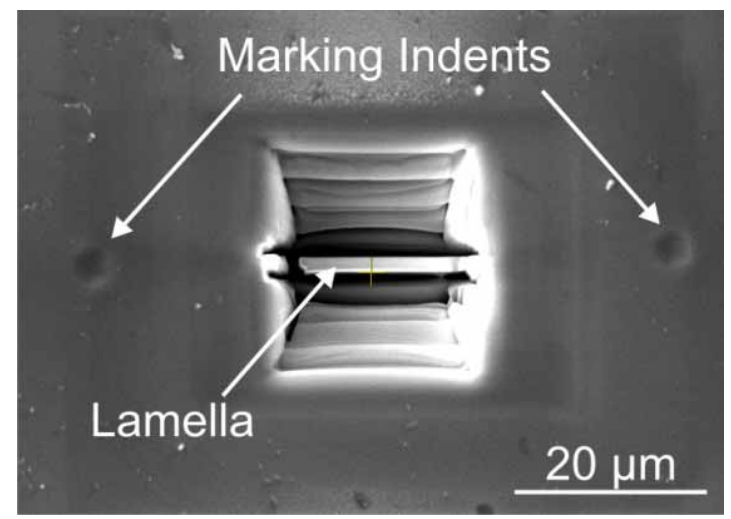

Fig. 2. SEM micrograph taken during the preparation process of the FIB lamella. To mark the location of the indented surface, the indents selected for subsequent microstructural analysis were placed between two marking indents.

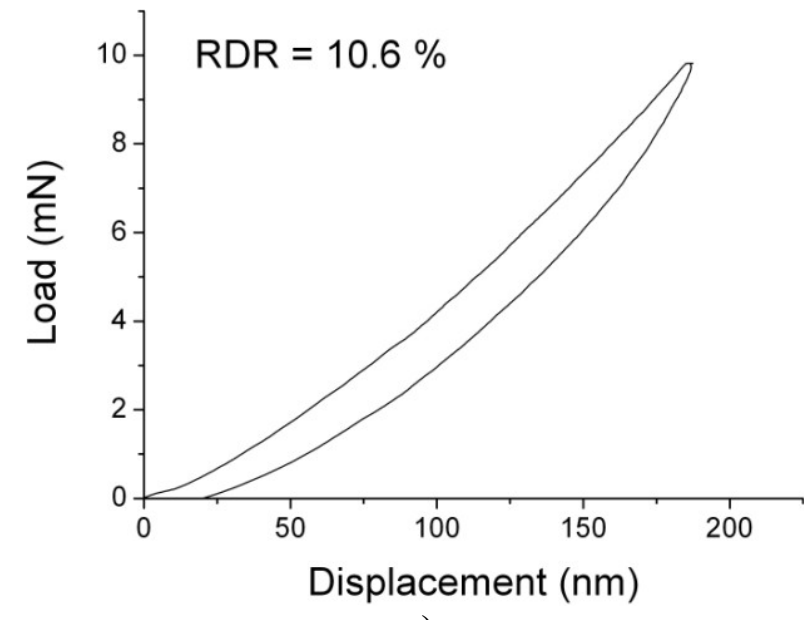

a)

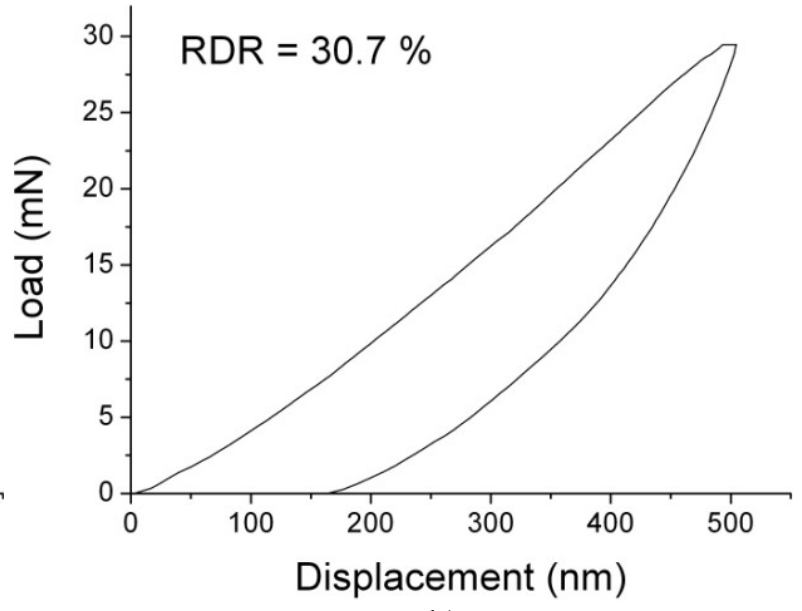

b)

Fig. 3. Load-displacement curves for nanoindentation with different maximum loads of a) $10 \mathrm{mN}$ and b) $30 \mathrm{mN}$.

In contrast, additional microstructural features can be observed below the $30 \mathrm{mN}$ indent, Fig. 5. The considerably deeper residual indent can even be observed in the bright-field image of the TEM lamella, Fig. 5a. The microstructure directly below the indented surface exhibits martensitic features as well as an increased dislocation density. The diffraction pattern of region 1 (circle 1 in Fig. 5a) shows only spots of the B2 phase, Fig. 5b. A detailed image of region 2 (circle 2 in Fig. 5a) is shown in Fig. 5c. The corresponding diffraction pattern (Fig. 5d) exhibits the splitting of spots (highlighted by two white arrows), which clearly indicates the presence of the B19' phase [24]. This result is also confirmed by additional spots of type [ $1 / 21 / 20]$. In summary, our detailed TEM observations demonstrate that, in qualitative agreement with our previous studies [25, 26], both plastic deformation and the stabilization of martensite below the indenter tip contribute to the deformation 
during loading and to the residual deformation after unloading when NiTi is indented with relatively large maximum loads. The microstructural results presented here highlight that various deformation processes contribute to the nanoindentation response of NiTi. Moreover, the relative effect of these processes depends on the maximum loads used during indentation. It is likely that the complex interaction between the stress-induced martensitic transformation, plastic deformation by dislocation slip and stabilization of martensite during unloading also depends on different testing temperatures and different microstructures. While further work is necessary to fully quantify the individual contributions of different deformation mechanisms as a function of these experimental parameters, the microstructural changes after indentation with different maximum loads can be at least qualitatively explained based on the results presented here: Nanoindentation is associated with multiaxial stress and strain states in the material below the indenter tip. The deformation below a spherical indenter tip (indenter radius $\mathrm{R}$ ) can be characterized by a representative strain $\varepsilon$ [27, 28]:

$$
\varepsilon=1.2 \frac{a}{R}
$$

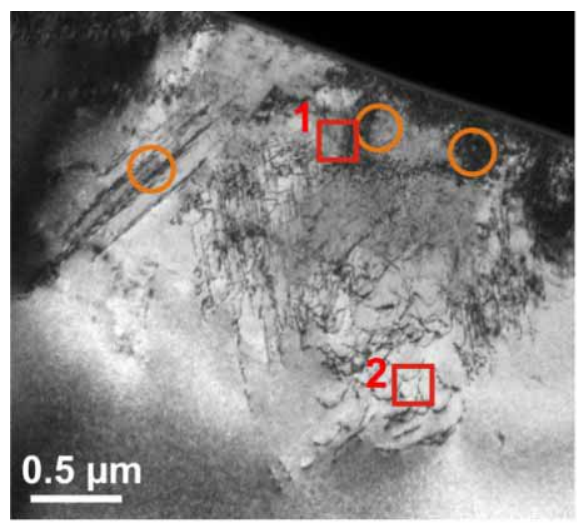

a)

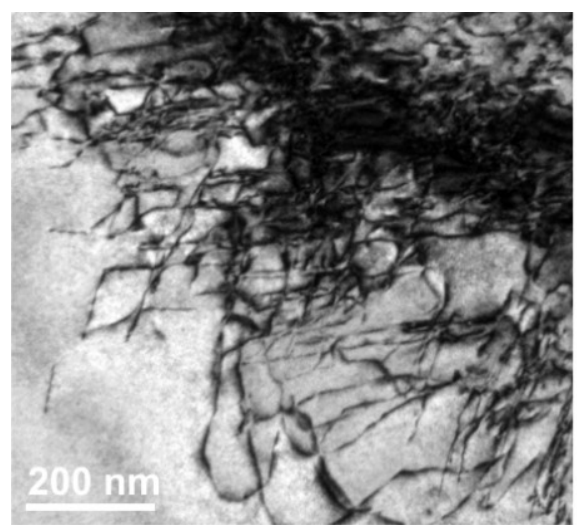

c)

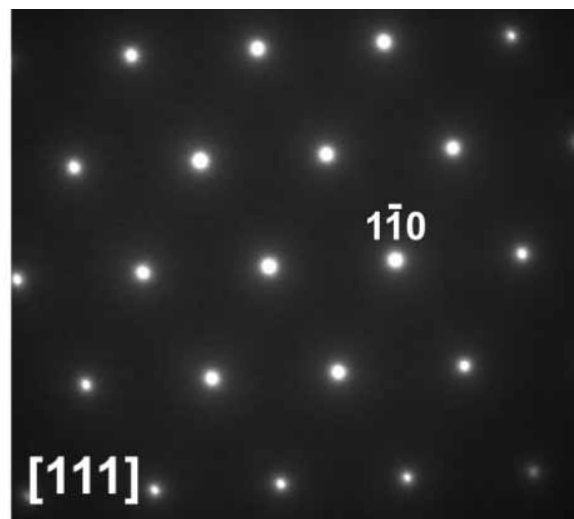

b)

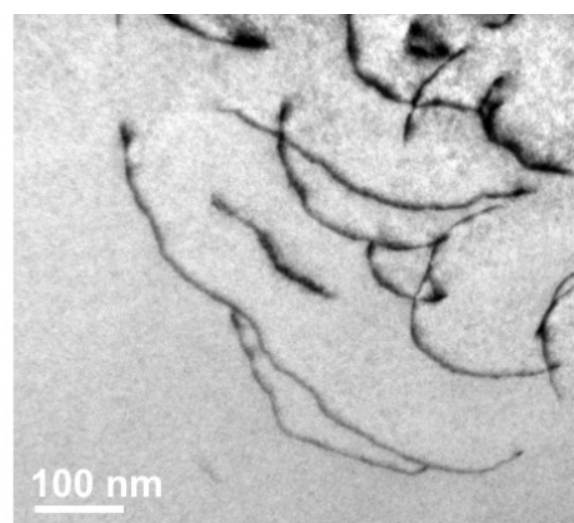

d)

Fig. 4. TEM observations below the indented surface for indentation with a maximum load of $10 \mathrm{mN}$. a) Bright field image of the region directly below the indent. Orange circles indicate regions where diffraction patterns were taken. Red boxes indicate regions where dislocations were documented in greater detail. b) Representative diffraction pattern (obtained after tilting the sample) of the regions marked in a). Spots of type [110] from the B2 phase are observed. $\mathrm{c}+\mathrm{d}$ ) Bright field images of dislocations c) at a distance of $\sim 200 \mathrm{~nm}$ (Box 1 in a)), and d) at a distance of $\sim 1.5 \mu \mathrm{m}$ (Box 2) from the indented surface.

As the contact radius, a, increases with increasing indentation depth [27], the representative strain associated with spherical indentation generally also increases. Therefore, higher maximum loads are associated with larger representative strains. In addition, our TEM observations show that the high strain gradients that are required below the indenter tip [28],

$$
\eta=\frac{1}{5 R},
$$


can not be provided by the stress-induced martensitic transformation alone. Instead, and in close analogy to the indentation response of conventional elasto-plastic materials, geometrically necessary dislocations must be formed and accumulated in the most deformed region [29]. As the strain gradient during spherical indentation is independent of the indentation depth (see equation (3)), even indentation experiments with small maximum loads result in an increasing density of dislocations below the indenter tip, see also Fig. 4.

Finally, we qualitatively consider the effect of an increasing dislocation density on the reverse transformation of stress-induced martensite during unloading. Fig. 6 schematically shows the microstructural evolution during nanoindentation experiments with different maximum loads. Indentation with a relatively small maximum load is associated with small strains (about $4.5 \%$ at the maximum indentation depth, as estimated from Eq. (2)). During loading, both the stress-induced martensitic transformation and plastic deformation contribute to these strains, Fig. 6a. During unloading, the reverse transformation occurs and an austenitic microstructure with an increased dislocation density is observed after complete unloading, Fig. 6b. Indentation with larger maximum loads $(30 \mathrm{mN})$ is associated with higher strains (about $6.5 \%$ ), Fig. 6c. The representative volume is larger than in the case of small maximum loads, and a larger number of dislocations is generated during loading even at greater distances from the surface (see also Figs. 4 and 5). Moreover, a larger volume of the material below the indenter tip is transformed into martensite, and plastic deformation contributes more strongly to the deformation

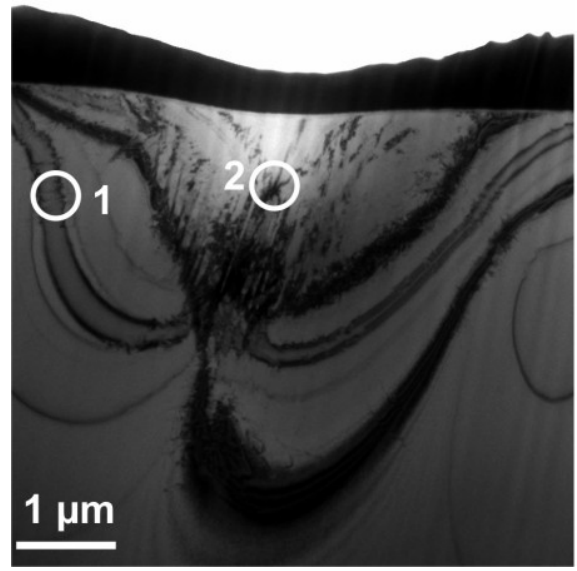

a)

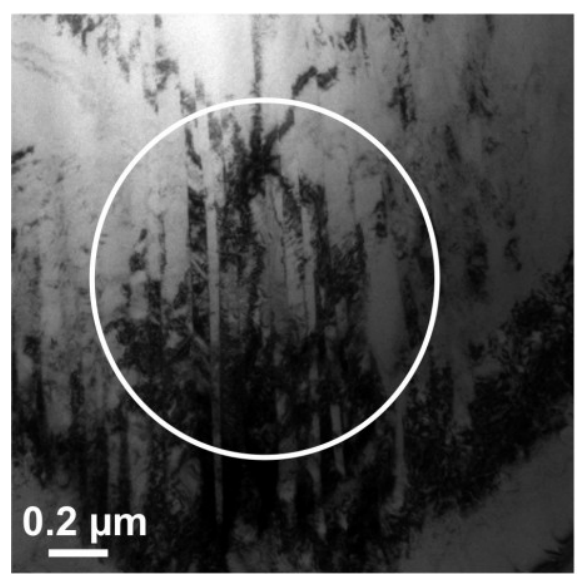

b)

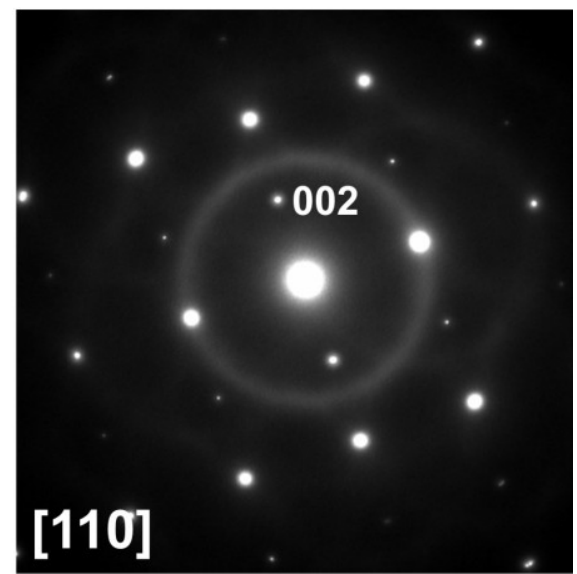

b)

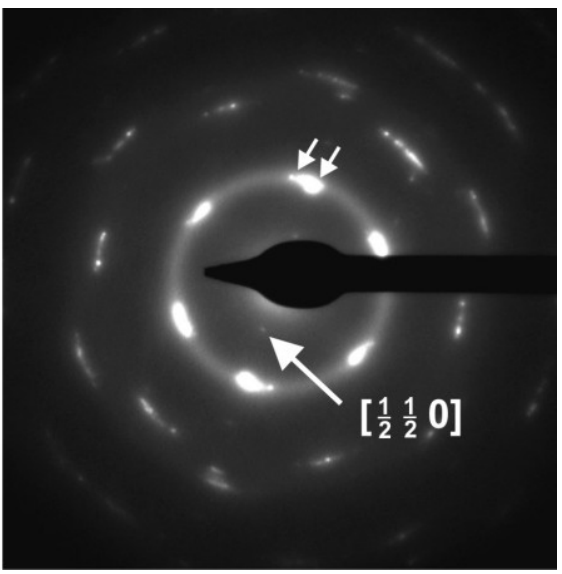

d)

Fig. 5. TEM observations below the indented surface (maximum load: $30 \mathrm{mN}$ ). a) The bright field image of the region below the indent shows both martensitic regions and dislocations. The regions marked by circles are presented in greater detail: b) Diffraction pattern of region 1 with spots of the B2 phase. $\mathrm{c}+\mathrm{d}$ ) The bright field image of region 2 and the corresponding diffraction pattern (which exhibits splitting of spots and spots of type $[1 / 21 / 20]$ ) indicate the presence of stabilized martensite. The diffraction patterns in b) and d) were obtained after tilting the sample.

directly below the surface of the NiTi specimen. During unloading (Fig. 6d), the higher dislocation density below the indenter tip impedes the reverse transformation to austenite; similar pinning processes of martensite have been observed under various loading conditions [30, 31]. In consequence, both dislocations and stabilized martensite can be observed after unloading, Fig. 6d (see also Fig. 5). 


\section{Summary and Conclusions}

We have investigated the microstructural evolution during spherical nanoindentation of pseudoelastic bulk NiTi by careful TEM observations of the deformed microstructures. Indentation experiments with small maximum loads are associated with small residual displacements after unloading. The indented microstructure after unloading is characterized by an austenitic matrix with few dislocations. Indentation with larger maximum loads results in a more pronounced remnant deformation. This residual deformation is related to a high dislocation density, which stabilizes martensite during unloading. These observations and our simple scenario relating dislocation slip and martensite stabilization demonstrate that the interaction of these microstructural deformation mechanisms plays an important role in determining the nanoindentation response of NiTi shape memory alloys.

maximum load

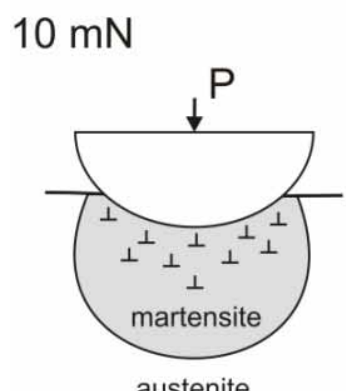

a)
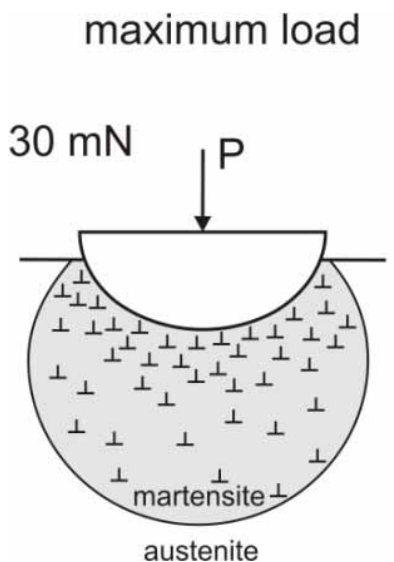

c)

\section{unload}

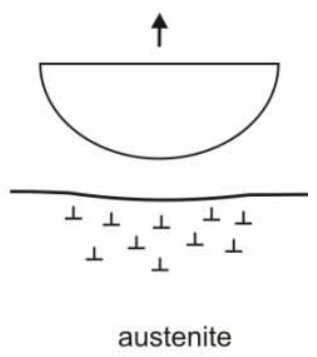

b)

\section{unload}

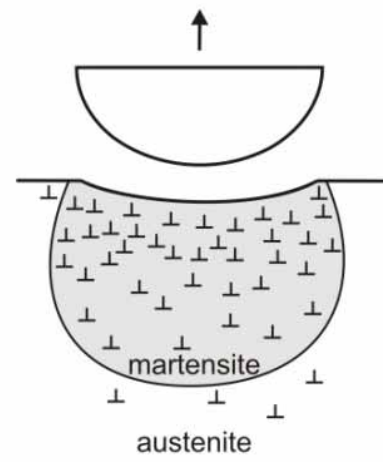

d)

Fig. 6. Schematic diagram of the microstructural evolution during nanoindentation experiments at maximum load and after unloading performed with $\mathrm{a}+\mathrm{b}) 10 \mathrm{mN}$ and $\mathrm{c}+\mathrm{d}$ ) $30 \mathrm{mN}$ maximum load. During loading both indentation loads result in stress-induced martensitic transformation and plastic deformation. During unloading the reverse transformation occurs for indentation with small loads, whereas indentation with larger loads results in higher dislocation density which impedes the reverse transformation to austenite.

\section{Acknowledgements}

This research was supported by the German Research Foundation (Deutsche Forschungsgemeinschaft, DFG) through projects A1 and A8 of the collaborative research center SFB459 ("Shape Memory Technology"). JP, AS and MW acknowledge funding in the framework of the Emmy Noether Program. 


\section{References}

[1] T. Duerig, A. Pelton and D. Stöckel, Mater. Sci. Eng. A 273-275, 149 (1999).

[2] M. Göken, M. Kempf and W. D. Nix, Acta Mater. 49, 903 (2001).

[3] G. Feng, W. D. Nix, Y. Yoon and C. J. Lee, J. Appl. Phys. 99, (2006).

[4] F. Haque, Surf. Eng. 19, 255 (2003).

[5] J. Y. Kim, S. H. Kim, J. S. Lee, K. W. Lee and D. Kwon, Metals Mater. Int. 12, 219 (2006).

[6] A. J. Muir Wood, J. H. You and T. W. Clyne, Smart Mat., Smart Sys. III: Proceedings of SPIE 5648216 (2004).

[7] A. J. Muir Wood, S. Sanjabi, Y. Q. Fu, Z. H. Barber and T. W. Clyne, Surf. Coat. Technol. 202, 31315 (2007).

[8] W. Ni, Y.-T. Cheng and D. S. Grummon, Surf. Coat. Technol. 177-178, 512 (2004).

[9] H.X. Zheng, J. Pfetzing, J. Frenzel, G. Eggeler, Int. J. Mater. Res 100, 594 (2009)

[10] C. P. Frick, T. W. Lang, K. Spark and K. Gall, Acta Mater. 54, 2223 (2006).

[11] W. M. Huang, J. F. Su, M. H. Hong and B. Yang, Scr. Mater. 53, 1055 (2005).

[12] W. C. Crone, G. A. Shaw, D. S. Stone, A. D. Johnson and A. B. Ellis, Soc. Exp.1 Mech. 1 (2003).

[13] G. A. Shaw, D. S. Stone, A. D. Johnson, A. B. Ellis and W. C. Crone, Appl. Phys. Lett. 83, 257 (2003).

[14] X. G. Ma and K. Komvopoulos, Appl. Phys. Lett. 83, 3773 (2003).

[15] X. G. Ma and K. Komvopoulos, Appl. Phys. Lett. 84, 4274 (2004).

[16] A. J. M. Wood and T. W. Clyne, Acta Mater. 54, 5607 (2006).

[17] A. J. M. Wood, S. Sanjabi, Y. Q. Fu, Z. H. Barber and T. W. Clyne, Surf. Coat. Technol. 202, 3115 (2008).

[18] M. Rester, C. Motz and R. Pippan, Acta Mater. 55, 6427 (2007).

[19] M. Rester, C. Motz and R. Pippan, Phil. Mag. 88, 879 (2008).

[20] H. Zheng, J. Rao, J. Pfetzing, J. Frenzel, C. Sornsen and G. Eggeler, Scr. Mater. 58, 743 (2008).

[21] S. R. Jian, Nanoscale Res. Lett. 3, 6 (2008).

[22] G. Abadias, A. Michel, C. Tromas, C. Jaouen and S. N. Dub, Surf. Coat. Technol. 202, 96 (2007).

[23] X. G. Ma and K. Komvopoulos, J. Mater. Res. 20, 1808 (2005).

[24] A. I. Lotkov, S. F. Dubinin, S. G. Teplouchov, V. N. Grishkov and V. P. Scorobogatov, J. Phys. IV 5, 551 (1995).

[25] J. Pfetzing, A. Schaefer, Ch. Somsen and M. F.-X. Wagner, Int. J. Mater. Res. accepted, (2009).

[26] J. Pfetzing, M. F.-X. Wagner, J. Frenzel, C. Somsen and G. Eggeler,"Thermomechanical constraints on pseudoelasticity during nanoindentation of binary and ternary $\mathrm{NiTi}(\mathrm{Fe})$ alloys" International Conference on Martensitic Transforamtion Santa Fe, 2008, edited by A. B. Saxenas (TMS, 2009).

[27] A. C. Fischer-Cripps: Nanoindentation (Springer, New York, USA, 2004).

[28] N. I. Tymiak, D. E. Kramer, D. F. Bahr, T. J. Wyrobek and W. W. Gerberich, Acta Mater. 49, 1021 (2001).

[29] W. D. Nix and H. J. Gao, J. Mech. Phys. Solids 46, 411 (1998).

[30] W. Tirry and D. Schryvers, Mater. Sci. Eng. A 481, 420 (2008).

[31] M. F. X. Wagner, N. Nayan and U. Ramamurty, J. Phys. D 41, (2008). 